\title{
Linkage disequilibrium narrows locus for venous malformation with glomus cells (VMGLOM) to a single $1.48 \mathrm{Mbp}$ YAC
}

\author{
Alexandre Irrthum ${ }^{1}$, Pascal Brouillard ${ }^{1}$, Odile Enjolras ${ }^{2}$, Neil F Gibbs ${ }^{3}$, \\ Lawrence F Eichenfield ${ }^{3}$, Bjorn R Olsen ${ }^{4}$, John B Mulliken ${ }^{5}$, Laurence M Boon ${ }^{1,6}$ and \\ Miikka Vikkula ${ }^{1}$
}

\begin{abstract}
${ }^{1}$ Laboratory of Human M olecular Genetics, Christian de Duve Institute of Cellular Pathology and Université catholique de Louvain, Brussels, Belgium; ${ }^{2}$ Department of Dermatology, Hôpital Lariboisière, Paris, France; ${ }^{3}$ Pediatric Dermatology, Children's Hospital San Diego, San Diego, CA; ${ }^{4}$ Department of Cell Biology, Harvard Medical School and Harvard-Forsyth Department of Oral Biology, Harvard School of Dental Medicine; ${ }^{5}$ Division of Plastic Surgery, Children's Hospital and Harvard Medical School, Boston, MA, USA; ${ }^{6}$ Center for Vascular Anomalies, Division of Plastic Surgery, Cliniques Universitaires St-Luc, Université catholique de Louvain, Brussels, Belgium
\end{abstract}

\begin{abstract}
Venous malformations with glomus cells are localised cutaneous lesions of vascular dysmorphogenesis. They are usually sporadic, but sometimes familial. Using five families, we mapped the locus, VMGLOM, to chromosome 1p21-p22. In order to refine this locus, spanning 4-6 Mbp, we then studied seven additional families. They exhibited linkage to VMGLOM and the combined lod score for all 12 families was 18.41 at $\theta=\mathbf{0 . 0}$ for marker D1S188. We found a distinct haplotype shared by seven families, comprising seven alleles which are rare in the general population $(P<0.01)$. This indicates that the haplotype is identical by descent in all seven families, and hence the locus can be refined by inferring ancestral crossovers. Using this approach, we position the causative gene between two markers on the same non-chimeric YAC of $1.48 \mathrm{M} \mathrm{bp}$, a feasible size for positional cloning. As there is no known gene involved in vasculogenesis and/or angiogenesis in this YAC, the identification of the causative gene is likely to reveal a novel regulator or vascular development. European Journal of Human Genetics (2001) 9, 34-38.
\end{abstract}

Keywords: vascular anomaly; haplotype sharing; glomangioma; multiple glomus tumor; chromosome 1p21-p22

\section{Introduction}

Vascular malformations are localised errors of morphogenesis. ${ }^{1}$ They are classified by the histologic appearance of the anomalous channels as either arterial, capillary, lymphatic, venous or combined malformations. ${ }^{2}$ Vascular lesions are usually cutaneous and often disfiguring. Functional and even life-threatening problems can occur if these anomalies involve deeper structures or viscera. Venous malformations (VMs) are the most common in referral centres. They appear

Correspondence: Miikka Vikkula, MD, PhD, Laboratory of Human Molecular Genetics, Christian de Duve Institute of Cellular Pathology \& Université catholique de Louvain, Avenue Hippocrate $75+4$, bp 75.39, B-1200 Brussels, Belgium. Tel: +32 2 764 6530; Fax: +32 2764 7548; E-mail: vikkula@bchm.ucl.ac.be

Received 3 April 2000; revised 17 August 2000; accepted 7 September 2000 as bluish cutaneous lesions that are localised, either small or extensive. $^{1}$

To identify genes involved in venous morphogenesis, we studied families with inherited cutaneous venous malformations (OMIM No 600195), and found the first genetic locus on chromosome9p21. ${ }^{3}$ Subsequently, we identified the causative activating R849W mutation in TIE2/TEK, a receptor tyrosine-kinase specifically expressed in endothelial cells. ${ }^{4}$ In addition to this locus, we discovered a second 4-6 cM locus on chromosome1p21-p22 that is linked to venous malformations in five families (VMGLOM, OMIM No 138000). ${ }^{5}$ Although the cutaneous lesions in these families clinically looked very similar to the 9p-linked VMs, histologic analysis of the lesions revealed the presence of 'glomus cells' around the lumens of the distorted vessels, hence the traditional histopathologic name 'glomangioma'. The causative gene is 
unknown and we are using positional cloning for its identification.

In order to refine the locus, we here report seven additional families with venous anomalies, show linkage to the VMGLOM locus and reveal the existence of a founder effect for some of the families, thereby refining the locus to the size of a single YAC.

\section{Material and methods}

The pedigrees of the seven new families with venous malformations with glomus cells are shown in Figure1. Each participant gave informed consent, as approved by the ethics committee of the medical faculty of the Université catholique de Louvain, Brussels, Belgium. Clinical history was taken and physical examination was performed on all family members participating in the study. Venous blood samples were drawn and DNA was extracted from the buffy coats (Qiagen QIAamp DNA Blood Mini Kit, Westburg, Leusden, The Netherlands). Histological characterisation was done on lesions excised from individuals in all families except family Al.

Genotyping of individuals was performed, as described elsewhere. ${ }^{3}$ We used microsatellite markers located in the VMGLOM region on the basis of CEPH (Le Centre d'Etude du

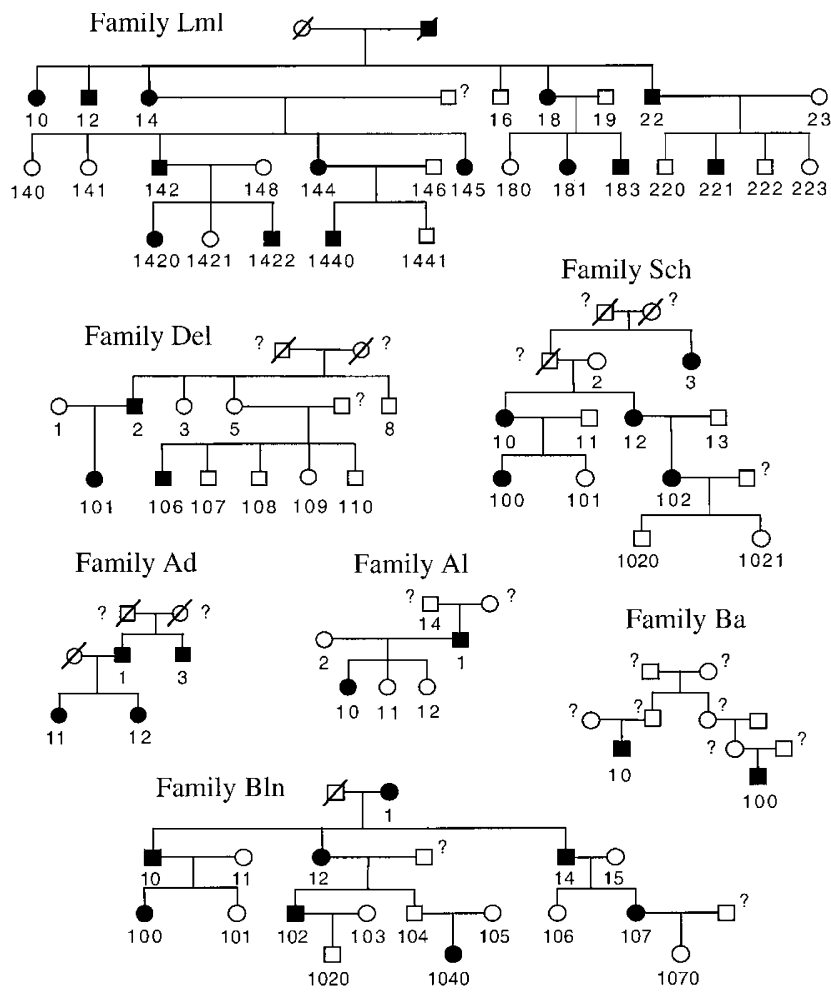

Figure 1 Pedigrees of seven families with venous malformations with glomus cells. Solid symbol: affected person; ?: unknown affection status; /: deceased person. Individuals with a number have been genotyped.
Polymorphisme Humain: http://www.cephb.fr/), CHLC (The Cooperative Human Linkage Center: http://chlcl.fccc.edu/ ChlcMarkerMaps.html) and Whitehead/MIT databases (The Whitehead Institute for Biomedical Research/MIT Center for Genome Research: http://www-genome.wi.mit.edu/). Marker D1S2849 gave mediocre results that were improved by replacing primer AFM350tg9m by primer 5'-ATTCCCTACCCTCCAGAC-3', giving a shorter PCR product. We also used novel CA repeat microsatellites that had been isolated in our laboratory as part of the construction of a physical map for the region (33CA1, 50CA1, 69CA1 and 75CA1). ${ }^{6}$ Calculations were performed using the MLINK program of the LINKAGE package, ${ }^{7}$ with parameters as described. ${ }^{5}$ Phenocopies were not allowed and three penetrance classes were defined on the basis of Iqbal et $\mathrm{al}^{8}$ (Table 1).

In order to detect putative haplotype sharing, three affected individuals from each family (except for families $\mathrm{Ba}$ and $A 1$, in which there are only two affected individuals) were genotyped for every available microsatellite marker in the VMGLOM locus (Figure2). The precise order of the markers was known by construction of the physical map of the locus. ${ }^{6}$ The radioactive PCR products for each marker were resolved on a separate polyacrylamide gel to allow consistent scoring of the alleles across the families. Absolute sizes of the alleles were determined by running a pUC18 plasmid sequencing reaction on the same gel. As an additional control for the sizes of the alleles, PCR reactions from CEPH reference individuals (Nos88415, 134702, 133101 and 133102, http://www.cephb.fr/cephdb/) were also included. To estimate the frequencies of the shared alleles and haplotypes in the general population, 16 triplets (father, mother, child), belonging to the genetically heterogeneous Belgian population, were also genotyped. The frequencies of the shared alleles from the first and second haplotypes were calculated in the affected families and in our control dataset, comprising 64 haplotypes. Statistical significance of the frequency bias was assessed using the uncorrected chi-square test of independence.

In order to retrieve from databases genomic sequences in the VM GLOM candidate region, the sequences of the markers

Table 1 Lod scores for marker D1S188 in the seven families, for disease-allele frequency 0.0001, 10 marker alleles with equal frequencies 0.1 , and three liability classes with penetrances $90 \%, 80 \%$ and $70 \%$, respectively, for individuals of age $\geq 16$ years, 12 years $\leq$ age $<16$ years, and $<12$ years

\begin{tabular}{lrrrrrrr}
\hline Family & $\theta=0.0$ & $\theta=0.01$ & $\theta=0.05$ & $\theta=0.1$ & $\theta=0.2$ & $\theta=0.3$ & $\theta=0.4$ \\
\hline Lml & 3.78 & 3.75 & 3.56 & 3.27 & 2.56 & 1.70 & 0.70 \\
Sch & 1.28 & 1.26 & 1.14 & 1.00 & 0.70 & 0.42 & 0.18 \\
Bln & 0.75 & 0.74 & 0.70 & 0.63 & 0.47 & 0.29 & 0.12 \\
Del & 0.56 & 0.54 & 0.47 & 0.39 & 0.23 & 0.09 & 0.02 \\
Ba & 0.32 & 0.31 & 0.26 & 0.20 & 0.11 & 0.05 & 0.02 \\
Al $^{\text {a }}$ & -0.18 & -0.15 & -0.06 & 0.01 & 0.08 & 0.09 & 0.07 \\
Ad $^{b}$ & -0.52 & -0.45 & -0.27 & -0.15 & -0.04 & -0.00 & -0.00 \\
\hline
\end{tabular}

ahistological diagnosis not known; ${ }^{\mathrm{b}}$ uninformative marker. 


\begin{tabular}{|c|c|c|c|c|c|c|c|c|c|c|}
\hline & \multicolumn{10}{|c|}{ HAPLOTYPE A } \\
\hline & $\begin{array}{c}\mathrm{BI} \\
\text { USA }\end{array}$ & $\begin{array}{c}\mathrm{Bt} \\
\mathrm{Bel}\end{array}$ & $\begin{array}{c}\text { Sh } \\
\text { USA }\end{array}$ & $\begin{array}{c}F \\
\text { Sco }\end{array}$ & $\begin{array}{c}\mathrm{T} \\
\text { Ita }\end{array}$ & $\begin{array}{l}\text { BIn } \\
\text { Fra }\end{array}$ & $\begin{array}{l}\text { Sch } \\
\text { Ger }\end{array}$ & $\begin{array}{l}\mathrm{n} / \mathrm{N} \\
\mathrm{fam} .\end{array}$ & $\begin{array}{l}\mathrm{n} / \mathrm{N} \\
\text { con. }\end{array}$ & $\mathbf{P}$ \\
\hline A205XD5 & 241 & $239 / R$ & $241 / 245$ & $245 / R$ & 241 & 241 & 245 & - & - & - \\
\hline D1S435 & 175 & 157 & 161 & 175 & 175 & 157 & 157 & $5 / 12$ & $25 / 60$ & 1.000 \\
\hline B337XE1 & 263 & 263 & 267 & 281 & 259 & 263 & 267 & $4 / 12$ & $24 / 54$ & 0.481 \\
\hline D1S188 & 162 & 168 & 166 & 166 & 166 & 168 & 166 & $3 / 12$ & $6 / 54$ & 0.205 \\
\hline D1S1170+ & 118 & 118 & 118 & 118 & 122 & 118 & 118 & $10 / 12$ & $30 / 60$ & 0.034 \\
\hline $33 \mathrm{CA} 1$ & 156 & 156 & 156 & 156 & 152 & 156 & 156 & $9 / 12$ & $18 / 62$ & 0.002 * \\
\hline D1S2804 & 179 & 179 & 179 & 179 & 179 & 179 & 179 & $7 / 12$ & $10 / 62$ & $0.001 *$ \\
\hline D1S424 & 223 & 225 & 223 & 223 & 223 & 225 & 223 & $5 / 12$ & $9 / 62$ & 0.033 \\
\hline D1S406+ & 200 & 200 & 200 & 204 & 200 & 196 & 200 & $5 / 12$ & $6 / 64$ & 0.003 * \\
\hline 69CA1 & 171 & $169 / 171$ & 171 & 171 & 171 & 171 & 171 & $9 / 12$ & $15 / 60$ & 0.001 * \\
\hline D1S2776 & 206 & 206 & 206 & 206 & 206 & 206 & 206 & $8 / 12$ & $8 / 62$ & $0.000 *$ \\
\hline $50 \mathrm{CA} 1$ & 127 & $125 / 127$ & 129 & 127 & 127 & 127 & 127 & $7 / 12$ & $2 / 48$ & $0.000 *$ \\
\hline D1S2868 & 146 & 146 & 146 & 146 & 146 & 146 & 146 & $7 / 12$ & $14 / 62$ & 0.012 \\
\hline 75CA1 & $171 / 173$ & $171 / 173$ & 173 & 173 & 171 & 173 & 173 & $6 / 12$ & $5 / 54$ & $0.001 *$ \\
\hline D1S2849 & 179 & 179 & 179 & 179 & 179 & 179 & 179 & $8 / 12$ & $17 / 64$ & $0.007^{\star}$ \\
\hline D1S2779 & 229 & 231 & 233 & 231 & 241 & 231 & 231 & $4 / 12$ & $21 / 62$ & 0.971 \\
\hline D1S236 & 194 & 210 & 194 & 190 & 212 & $190 / R$ & $190 / R$ & $3 / 12$ & $37 / 62$ & 0.027 \\
\hline D1S2775 & 201/R & $199 / 201$ & 201 & 201 & 201 & $195 / R$ & $201 / \mathrm{R}$ & - & - & - \\
\hline
\end{tabular}

\begin{tabular}{|c|c|c|c|c|c|c|c|c|}
\hline & \multicolumn{7}{|c|}{ HAPLOTYPE B } & \multirow[b]{2}{*}{$\begin{array}{l}\text { Lml } \\
\text { Fra }\end{array}$} \\
\hline & $\begin{array}{c}\text { Al } \\
\text { USA }\end{array}$ & $\begin{array}{c}\text { Ba } \\
\text { Yug }\end{array}$ & $\begin{array}{l}\text { Del } \\
\text { Bel }\end{array}$ & $\begin{array}{l}\text { Ad } \\
\text { Fra }\end{array}$ & $\begin{array}{l}\mathrm{n} / \mathrm{N} \\
\text { fam. }\end{array}$ & $\begin{array}{l}n / N \\
\text { con. }\end{array}$ & $\mathbf{P}$ & \\
\hline A205XD5 & 241 & 241 & 241 & 241 & - & - & - & $241 / R$ \\
\hline D1S435 & $157 / 161$ & 161 & 175 & $157 / 161$ & - & - & - & $161 / \mathrm{R}$ \\
\hline B337XE1 & 259 & $259 / 267$ & 259 & 259 & $5 / 12$ & $14 / 54$ & 0.276 & $263 / R$ \\
\hline D1S188 & 154 & 160 & 154 & 152 & $2 / 12$ & $6 / 54$ & 0.594 & 168 \\
\hline D1S1170+ & 118 & $118 / 122$ & $118 / 122$ & 122 & $10 / 12$ & $30 / 60$ & 0.034 & 118 \\
\hline $33 \mathrm{CA} 1$ & 156 & 156 & 156 & 152 & $9 / 12$ & $18 / 62$ & 0.002 * & 142 \\
\hline D1S2804 & 185 & 185 & 183 & 185 & $3 / 12$ & $13 / 62$ & 0.756 & 191 \\
\hline D1S424 & 209 & 209 & 209 & 209 & $4 / 12$ & $28 / 60$ & 0.396 & 225 \\
\hline D1S406+ & 208 & $204 / 208$ & 208 & 208 & $5 / 12$ & $17 / 64$ & 0.290 & 208 \\
\hline 69CA1 & 169 & 169 & 171 & $169 / 171$ & $4 / 12$ & $30 / 60$ & 0.291 & 173 \\
\hline D1S2776 & 198 & 198 & 198 & 198 & $4 / 12$ & $11 / 62$ & 0.219 & 206 \\
\hline $50 C A 1$ & 125 & 125 & 125 & 127 & $5 / 12$ & $27 / 48$ & 0.365 & 125 \\
\hline D1S2868 & 148 & 148 & 148 & 148 & $4 / 12$ & $25 / 62$ & 0.650 & 144 \\
\hline $75 \mathrm{CA} 1$ & 171 & 171 & 171 & 175 & $7 / 12$ & $36 / 54$ & 0.584 & 171 \\
\hline D1S2849 & 181 & 179 & 181 & 183 & 2/12 & $10 / 64$ & 0.928 & 176 \\
\hline D1S2779 & 235 & 233 & 235 & 241 & 2/12 & $3 / 62$ & 0.135 & 229 \\
\hline D1S236 & 194 & 212 & 210 & 212 & - & - & - & 212 \\
\hline D1S2775 & 201 & 201 & 199/201 & 199/201 & - & - & - & 199 \\
\hline
\end{tabular}

Figure 2 Haplotypes A and B sharing in VM GLOM. Numbers indicate sizes of alleles that segregate with the disorder in each family. At the top, symbol and geographic origin of family. USA: The United States of America; Bel: Belgium; Sco: Scotland; Ita: Italy; Fra: France; Ger: Germany; Yug: Yugoslavia. +: tetranucleotide repeat microsatellite. R: a recombinant individual in the family for this marker; X/Y: data not informative for linked allele. Marker alleles with a probable ancestral mutation differ from shared haplotype: white background; $\mathrm{n} / \mathrm{N}$ : number of the shared allele on total number of alleles; fam: families linked to VM GLOM; con: control individuals from the Belgian population. P: P-value for the uncorrected chi-square test in a $2 \times 2$ table; *: significant P-value $(P<0.01)$.

were compared to the human high-throughput genomic sequences using the blast sequence similarity search tool (http://www.ncbi.nlm.nih.gov/BLAST/). The ordering of the PAC clones containing the markers was visualised using the
Entrez Map Viewer tool (http://www.ncbi.nlm.nih.gov:80/ cgi-bin/Entrez/hum_srch?chr=hum_chr.inf\&query). Known genes and putative genes in the region were identified by comparing the unfinished PAC sequences to the non- 
redundant GenBank database using the NCBI blast tool, and to a database of EST contigs at the Swiss EM Bnet server (http:/ www.ch.embnet.org/software/aBLAST.html).

\section{Results}

Physical examination of the patients from the families with venous malformations with glomus cells revealed that the locations, number per individual and histological appearance of the lesions were similar to those documented in the five families initially reported. ${ }^{5}$

The number of affected males and females in the 12 families was 35 and 40 , respectively, and 59\% (26/44) of children of an affected parent had the disorder. Examination of the seven new pedigrees revealed that the condition skipped a generation twice (individuals Del5 and BIn104, Figure1). The autosomal dominant pattern of inheritance with high penetrance and the absence of sex bias are consistent with our previous data. $^{5}$

None of the seven new families formally excluded linkage to the VMGLOM locus. Lod scores for marker D1S188 are given for different recombination fractions in Table1. Family Lml showed strong linkage to the VM GLOM locus (lod score 4.05 for marker D1S2804). Families Sch, Bln, Del, Ad and Ba also showed evidence for linkage in the locus, with highest lod scores (at $\theta=0.0$ ) of 1.69 for marker D1S2776 (family Sch), 0.75 for marker D1S188 (family BIn), 0.56 for marker D1S188 (family Del), 0.56 for marker D1S2776 (family Ad), and 0.52 for marker D1S2776 (family Ba). Family A1 gave slightly negative lod scores (-0.18 for marker D1S188). The maximum combined lod score for the seven new families was $5.99(\theta=0.0)$ for marker D1S188, and for all 12 families it was $18.41(\theta=0.0)$, with no obvious evidence for locus heterogeneity. However, four young unaffected recombinants were observed (Lml223, A112, Sch1020 and Bln1070, aged 14 years, 10 years, 2 years and 1 year, respectively).

Haplotypic analysis of the seven families defined new obligatory recombinations in VMGLOM between markers AFMB337XE1 and D1S188 (affected individual LmI22) on the telomeric end of VMGLOM and between markers D1S236 and D1S2779 (affected individuals Sch3 and BIn100, and unaffected individual BIn 1020) in the centromeric region. These findings narrow the locus by $2 \mathrm{CM}$ from AFMA205XD5D1S236 [Boon, 1999 No 811] to AFMB337XE1-D1S236, a region of about $3 \mathrm{cM}$ (on the basis of the Whitehead/MIT physical map). Comparison of the haplotypes linked to the condition in the 12 families revealed two distinct haplotypes, $A$ and $B$, shared by seven families (Bl, Bt, Sh, F, T, Bln, Sch), and by four families (Al, Ba, Del, Ad), respectively (Figure2). Family $\mathrm{Lml}$ had a unique haplotype.

Statistical significance of the apparent linkage disequilibrium was assessed using the chi-square independence test. The frequency bias is significant $(P<0.01)$ for seven out of the nine markers in the core of the first haplotype (between D1S2804 and D1S2849, Figure2, top). This supports the hypothesis of a founder effect for this haplotype, and allows us to refine the locus further by inferring ancestral recombinations. In contrast, alleles of the second haplotype do not show statistically significant enrichment from the general population, and thus the second haplotype is probably due to co-occurrence of frequent alleles by chance (Figure2, bottom). Thus, based on apparent ancestral crossovers in family $T$ for the first haplotype, the VMGLOM locus can be delineated between marker 33CA1 and marker D1S2779. These two markers, and all intervening markers, have been localised on the same non-chimeric $1.48 \mathrm{Mbp}$ YAC 957D9 (Whitehead/MIT database). ${ }^{6}$

In this $1.48 \mathrm{Mbp}$ VMGLOM region between 33CA1 and D1S2779, 13PACs with unfinished sequences were identified as they contained at least one of the markers in the interval. Moreover, two PACs with finished sequences were also identified as belonging to the same PAC contig. On the basis of the Entrez Genome Map Viewer, most of the region appears to be covered by unfinished sequences, although there are two gaps. The ordering of the markers is consistent with our data. ${ }^{6}$ In addition to putative genes and EST clusters, nine known or highly similar to known genes were identified in the region using sequence similarity searches. Known genes are EVI5 (ecotropic viral integration site5), FAP48 (FKBP-associated protein), GFI1 (growth factor-independent 1) and RPL5 (ribosomal protein L5). The five other genes are highly similar ( $>90 \%$ identity) to ACTG1 (actin, gamma 1), AIF1 (allograft inflammatory factor 1), BTK (Bruton agammaglobulinemia tyrosine kinase), HMG4 (highmobility group protein 4) and RPL29 (ribosomal protein L29).

\section{Discussion}

In this study, we described seven new families with inherited cutaneous venous malformations with glomus cells that showed linkage to the VMGLOM locus on chromosome 1p21-p22. By combining thelinkage data from these families with our previous data, the maximum two-point lod score was 18.41 (marker D1S188, $\theta=0.0$ ). The disease showed autosomal dominant inheritance pattern with high penetrance. Four young children were unaffected carriers. This non-penetrance was expected since it has been shown that the onset of the disease can be pubertal. ${ }^{5}$ In addition, unaffected individual Del5, with an affected son, had inherited the haplotype associated with the condition in her family and is thus likely to be a carrier. Individual BIn104, on the contrary, had not inherited the haplotype linked with the disorder in his family, and thus his daughter, Bln1040, who has a single small ventral lesion, is probably a phenocopy. This underscores the uncertainty of clinical diagnosis of single small cutaneous lesions in genetic studies of vascular anomalies. Taken together, our data suggest that the VMGLOM locus contains the major gene for venous malformations with glomus cells. 
Haplotypic analysis revealed recombinative events in affected individuals, narrowing the region from 5-6 cM to $3 \mathrm{cM}$. Furthermore, in a subset of the families, we discovered a haplotype that is significantly associated with the disease, underlining the existence of a founder effect. This linkage disequilibrium allowed us to further diminish the locus by inferring ancestral crossovers to the region between markers 33CA1 and D1S2779. These markers are contained in a single $1.48 \mathrm{Mbp} Y A C$, and thus this finding constitutes an important step towards positional cloning of the VMGLOM gene. Naturally, we cannot rule out the possibility that the apparent crossovers in markers 33CA1 and D 1 S1170 in family $\mathrm{T}$ are actually the consequence of marker mutations, as such mutations were observed inside the core of the first haplotype for markers D1S424, D1S406, 50CA1 and 75CA1 (Figure2, top). Taking this possibility into consideration, a very conservative analysis of the data delineates the locus between markers D1S188 and D1S2779.

Delineation of the region for the VMGLOM gene between markers 33CA1 and D1S2779 confirms our previous exclusion of several genes localised to the initial VMGLOM Iocus, either on the basis of mutation screening, for DR1 (depression of transcription 1), TFA (tissue factor) and TGRBR3 (transforming growth factor- $\beta$ receptor, type 3 ), or on the basis of function, for ABCR (ATP-binding-cassette transporter) and GLCLR ( $\gamma$-glutamylcysteine synthetase). ${ }^{5}$ Thanks to the human genome draft sequence, the VMGLOM region now contains nine genes of known or highly similar to known function. However, it is difficult to highlight the best candidate gene or to formally exclude others on the basis of current knowledge.

Identification of the VMGLOM gene will probably enhance understanding of the etiopathogenesis of venous malformations, with and without glomus cells. As mutations in tyrosine-kinase receptors and other signalling molecules important for angiogenesis and vasculogenesis are the causes for vascular dysmorphogenesis in man and genetically engineered mice, it will be of great interest to know if the VMGLOM gene is related to these pathways, and in particular, to TIE2/TEK signalling.

\section{Acknowledgements}

The authors thank all families for their invaluable participation. We also thank $\mathrm{Dr}$ Matt Warman for invaluable comments and suggestions. Studies from the authors' laboratories were supported, in part, by Organogenesis Inc, and the National Institutes of Health, HL33014 and AR36820 (to BRO), and Fonds de Développement Scientifique - Université cathol ique de Louvain, FNRS (Fonds National de la Recherche Scientifique), the Bel gian Federal Service for Scientific, Technical and Cultural Affairs and European Commission, ERB4001GT963858 (to MV). A Irrthum and P Brouillard are supported by fellowships from FRIA (Fonds pour la formation à la recherche dans l'industrie et dans I'agriculture). We are also grateful to Ms Ana Gutierrez for expert technical assistance.

\section{References}

1 Vikkula M, Boon LM, Mulliken JB, Olsen BR: Molecular basis of vascular anomalies. Trends Cardiovasc Med 1998; 8: 281-292.

2 Mulliken JB, Glowacki J: Hemangiomas and vascular malformations in infants and children: a classification based on endothelial characteristics. Plast Reconstr Surg 1982; 69: 412-422.

3 Boon LM, Mulliken JB, Vikkula M et al: Assignment of a locus for dominantly inherited venous malformations to chromosome9p. Hum Mol Genet 1994; 3: 1583-1587.

4 Vikkula M, Boon LM, Carraway KLI et al: Vascular dysmorphogene sis caused by an activating mutation in the receptor tyrosine kinase TIE2. Cell 1996; 87: 1181-190.

5 Boon LM, Brouillard P, Irrthum A et al: A gene for inherited cutaneous venous anomalies ('glomangiomas') localizes to chromosome1p21-22. Am J Hum Genet 1999; 65: 125-133.

6 Brouillard P, Olsen BR, Vikkula M: High-resolution physical and transcript map of the locus for venous malformations with glomus cells (V M GLOM ) on chromosome1p21-p22. Genomics 2000; 67(1): 96-101.

7 Lathrop GM, Lalouel JM, Julier C, Ott J: Strategies for multilocus linkage analysis in humans. Proc Natl Acad Sci USA 1984; 81: 3443-3446.

8 Iqbal A, Cormack GC, Scerri G: Hereditary multiple glomangiomas. Br J Plast Surg 1998; 51: 32-37. 